\title{
CTP: Phosphocholine Cytidyltransferase Alpha (CCT $\alpha)$ siRNA Induce Cell Death of Lung Cancer Cells
}

\author{
Rukia Marijani and Barack O. Abonyo*
}

Florida A \& M University, College of Pharmacy and Pharmaceutical Sciences Tallahassee, FL 32307

\begin{abstract}
CTP: Choline cytidylyltransferase (CCT) is a rate limiting enzyme required for the synthesis of phosphatidylcholine (PC) through the CDP-choline pathway, which is critical for cell proliferation. Cells conditionally lacking CCTa activity undergo death by apoptosis. Inhibition of CCTa expression or activity in cancer cells using current molecular techniques would provide an excellent non toxic mechanism of killing cancer cells. The purpose of this study was to investigate the possibility of using CCTa siRNA sequences as anticancer agents by studying their ability to induce death of lung cancer cells. p53 positive, A549 and negative, H1299 lung epithelial cell lines were transfected with various concentrations $(0-800 \mathrm{nM})$ of CCTa siRNA sequences $(\mathrm{A}, \mathrm{B}$, and $\mathrm{C}$ ) and scrambled sequence siRNA (siRNA S) as control for $24 \mathrm{hrs}$. Inhibition of CCTa expression by various siRNA was determined by Western blot analysis. Olympus microscope was used to observe the impact of CCTa knockdown on cell structure and morphology while the role of CCTa siRNA in cell toxicity and survival was analyzed by Cell Titer Blue (CTB) and Lactose dehydrogenase (LDH) assays. All the CCTa siRNA sequences significantly inhibited CCTa protein expression. Pictures captured by the microscope depicted that untreated and siRNA S treated cells were intact and had visible nuclei while CCTasiRNA transfected cells were shrunken with nuclei containing dark granular structures similar to apoptotic bodies. At higher CCTa-siRNA concentrations the cell membranes were completely degraded showing signs of cell lyses. CTB assay revealed that untreated cells exhibited high fluorescence intensity indicating presence of more viable cells, which decreased as CCTa-siRNA concentration increased. LDH assay showed that CCTa-siRNA transfected cells exhibited higher absorbencies than the untreated reflective of dead cells. Both assays indicated that siRNA $S$ treated cells exhibited results similar to untreated cells, suggesting that the cells death was specific for CCTasiRNA inhibition. Furthermore, addition of PC did not improve viability of CCTa-siRNA treated cells. These results demonstrate that CCTa-siRNAs knocked down CCTa protein resulting in cell death implying that the sequences could be useful as anticancer agents.
\end{abstract}

Keywords: CCT $\alpha$; siRNA; Phosphatidylcholine (PC); apoptosis

\section{Introduction}

The synthesis of phosphatidylcholine (PC) through the CDPcholine pathway is a crucial process in mammalian cells development, as PC has a critical role in membrane structure and cellular signaling [1]. Certain cancers are associated with changes in expression of genes and proteins involved in the replenishing the membranes through synthesis of the phosphatidylcholine (PC). Consistently elevated PC levels have been observed in several types of cancers including human breast cancer cells in culture [2,3]. Indeed, progression of cells from G0 to G1 is accompanied by an increased rate of PC synthesis [4]. van der Sanden et al. [5] reported that inhibition of PC synthesis induced expression of the endoplasmic reticulum stress and apoptosis-related protein CCAAT/enhancer-binding protein-homologous protein (CHOP/GADD153) leading to programmed cell death. Because PC biosynthesis is very much connected to cancer development, several drugs have been designed to inhibit its biosynthetic pathway. For instance, the inhibition of PC synthesis both in vivo and in vitro by the antitumor phospholipid analogs, ET-18-OCH3 (or edelfosine) and hexadecylphosphocholine (or miltefosine) correlates with their cytotoxic properties [6]. The observations that choline deficiency induces apoptosis and that PC supplementation does not rescue cells from apoptosis in the presence of antineoplastic phospholipids [7] strongly supports the hypothesis that sufficient amount of PC is required for cell proliferation including that of cancerous cells.

The limiting enzyme in the PC synthesis is CTP: Phosphocholine Cytidylyltransferase (CCT), which is derived from two genes on distinct chromosomes in human and mouse. Phosphate cytidylyltransferase 1 alpha (Pcytla) gene encodes the CCTa isoform and Phosphate cytidylyltransferase 1 beta (Pcyt1b) gene encodes the CCT $\beta 1$ (in human only), $\beta 2$, and $\beta 3$ isoforms [8]. The CCT $\alpha$ and CCT $\beta$ proteins are similar in several ways, for instance they have nearly identical amino acid sequences in the catalytic domain (residues $72-233$ for both), which is highly conserved among all CCTs from yeast to mammals and contains two motifs, NTP binding motif (HXGH) and RTEGISTS, which are involved in the binding of CTP to membranes [9-11]. Additionally, they have an almost identical membrane interaction domain (residues 256-288), which consists of three consecutive 11-residue repeats that form an amphipathic $\alpha$-helix that regulates CCT activity $[12,13]$. On the contrary, the amino terminus of CCT $\beta$ completely differs that of CCTa and does not include a nuclear localization sequence as CCTa. Two CCT $\beta$ transcripts are expressed in human tissues, ССТ $\beta 1$ is a smaller protein that lacks the carboxyl terminal domain while CCT $\beta 2$ has carboxyl terminus [14]. The carboxyl terminal domain of CCTa and $\beta 2$ are distinct, opening the possibility that the proteins are differentially regulated by anionic lipids and/or phosphorylation. CCT $\alpha$

*Corresponding author: Barack O. Abonyo, Florida A \& M University, College of Arts and Sciences, Department of Biological Sciences, Tallahassee, FL 32307 Telephone: 850-561-2553; FAX: 850-599-3347; E-mail: abonyo.barack@famu. edu, luo31@hotmail.com

Received March 10, 2011; Accepted March 30, 2011; Published May 21, 2011

Citation: Marijani R, Abonyo BO (2011) CTP: Phosphocholine Cytidyltransferase Alpha (CCTa) siRNA Induce Cell Death of Lung Cancer Cells. Pharm Anal Acta 2:121. doi:10.4172/2153-2435.1000121

Copyright: @ 2011 Marijani R, et al. This is an open-access article distributed unde the terms of the Creative Commons Attribution License, which permits unrestricted use, distribution, and reproduction in any medium, provided the original author and source are credited. 
has four functional domains: the amino terminal region (residues 1-71) contains a cluster of positively charged amino acids that specify nuclear localization $[12,15,16]$. The phospholipid sensor domain CCTa is phosphorylated exclusively on serine residues and the carboxyl terminal region (residues 315-367) contains all the phosphorylation sites. Increased phosphorylation attenuates the activation of CCT $\alpha$ by lipid mediators [17] and releases CCTa from the membrane [12].

CCTa has been reported to be highly essential for animal survival. Wang et al. [12] reported that deletion of exons 5 and 6 in the Pcytla gene resulted in zygotes that failed to form blastocysts, did not develop past embryonic day 3.5 (E3.5), and failed to implant. Knock out of CCT $\beta$ on the other hand resulted in mice with gonad degeneration and reproductive deficiency only [18]. Deletion of any of the CCT domains affects its function in one way or the other resulting in reduced PC synthesis. Removal of both the helical and carboxyl terminal domains gives rise to a dysregulated CCTa that has higher basal activity in the absence of lipid and is refractory to lipid stimulation (for review, see Wang et al. [12]) while removal of the entire CCT C-terminus results in an enzyme that retains catalytic activity at high CTP concentrations but cannot respond to lipid regulation [20,21]. Surprisingly, CCT protein that lacks the helical domain but retained the carboxyl terminal domain exhibits the same activities as full-length CCT in the absence and presence of lipid, suggesting that the last 57 residues of the carboxyl-terminus also regulated lipid responsiveness [22]. The domain specificity of CCT may be useful in designing molecular agents that targets CCT function for the purposes of inhibiting processes requiring functional CCT.

Functional CCT is essential for cancer progression. CCT transcription has been shown to be stimulated 2-fold during the $\mathrm{S}$ phase in alveolar type II epithelial cells [23]. Lack of functional CCT leads to cell death by either apoptosis or necrosis. Van der Sanden et al. [5] demonstrated that exposing MT58 (a mutant Chinese hamster ovary $(\mathrm{CHO})$ cell line containing a thermo-sensitive mutation in CCT) to a non-permissive temperature of $40^{\circ} \mathrm{C}$ resulted in $\mathrm{PC}$ depletion via an inhibition of CCT within $24 \mathrm{hrs}$ and resulted in apoptosis within 48 hrs. Chemical inhibition of CCT activity leads to cell death by apoptosis or paraptosis [24,25]. These reports help advance the theory that mammalian cells cannot survive in the absence of CCT especially CCTa and therefore designing molecular mechanisms of inhibiting its function may be useful in shutting down the progression of a variety of cancers. In this study, we hypothesized that various sequences of CCT $\alpha$-siRNA have the capacity to knockdown CCT expression and induce cell death in lung cancer cell lines. Herein, we report that CCTa siRNA sequences specifically inhibited CCTa protein expression resulting in cell death by apoptosis and necrosis. This death was not recoverable through addition of PC indicating that knockdown of CCT commit cells to their death possibly through many other mechanisms. The outcome of these experiments provides insightful information that could be used to develop CCT $\alpha$-siRNA as anticancer agents.

\section{Materials and Methods}

\section{Materials}

A549 and H1299 lung epithelial cell lines were purchased from American Type Culture Collection (ATCC, Manassas, VA). Three sequences ( $\mathrm{A}, \mathrm{B}$, and $\mathrm{C})$ of CCTa siRNA and scrambled sequence siRNA (siRNA S) were purchased from Invitrogen (Carlsbad, CA). Cell Titer Blue (CTB) and Lactose Dehydrogenase (LDH) assay kits were purchased from Promega (Madison, WI). All other chemicals and reagents were of pharmaceutical grade.

\section{Cell culture and treatment}

A549 and H1299 cells were grown in RPMI/F-12K and RPMI media, respectively; supplemented with $10 \%$ fetal calf serum, penicillin $(100 \mathrm{U} / \mathrm{ml})$, and streptomycin $(100 \mu \mathrm{g} / \mathrm{ml})$ in a humidified atmosphere of $5 \%$ carbon dioxide at $37^{\circ} \mathrm{C}$. Trypsin/EDTA- or PBS/EDTA-dispersed cells were suspended in fresh medium in flasks at $0.2 \times 10^{6}$. Experiments were performed after subcultured cells reached $\sim 80 \%$ confluence.

\section{Western immunoblotting of CCT $\alpha$ protein}

A549 cells cultured to $80 \%$ confluence were transfected with CCT $\alpha$-siRNA A, B and C (0-800 nM), preincubated with FuGENE 6 transfection reagent (Roche diagnostics) at room temperature. After 24 $\mathrm{hr}$ incubation, cell suspensions were centrifuged at $1000 \mathrm{rpm}$ and cell pellets resuspended in lysis buffer (20 mM Tris, $\mathrm{pH} 7.4,2 \mathrm{mM}$ EDTA, $150 \mathrm{mM} \mathrm{NaCl}, 0.5 \%$ Triton X-100 and one tablet $/ 10 \mathrm{ml}$ of protease inhibitor cocktail). Samples were sonicated for $3 \mathrm{~s}$, centrifuged (5 min, RT at $1000 \mathrm{rpm}$ ) and supernatants collected for total protein content analysis. Cell lysates were separated by electrophoresis on $10 \%$ SDS-polyacrylamide gels (50 $\mathrm{gg}$ protein/lane) and then transferred to Immobilon-P PVDF membranes. Blots were blocked at $4^{\circ} \mathrm{C}$ overnight in 5\% Carnation Instant Milk in PBS containing 0.05\% Tween 20 (PBST) and then incubated overnight at $4^{\circ} \mathrm{C}$ with $5 \mu \mathrm{g} / \mathrm{ml}$ mouse antihuman CCT $a$ affinity purified antibody. Membranes were washed three times with PBST, incubated with 1:1500 goat anti-mouse IgG-horse radish peroxidase in PBST for 6 hrs then washed three times in PBST. Immunoblot images were obtained using a Flour-s Max Multimager.

\section{Cell viability and toxicity assays}

A549 and H1299 cells cultured to 80\% confluence were transfected with CCTa siRNA A, B and C and siRNA S (0-800 nM) alone and plus Phosphatidylycholine $(100 \mu \mathrm{M})$ in serum free media, preincubated with FuGENE 6 (Roche diagnostics) at room temperature as indicated above. Cell images were visualized and captured by an Olympus fluorescent microscope (40X objective) fitted with an Olympus DP70 camera. Images were documented using Adobe Photoshop. To determine concentration dependent cell viability, A549 cells were treated with different concentrations of CCTa-siRNA and siRNA S in 96 well plates and living or dead cells detected by CTB or LDH cell viability assays, respectively. 10,000 cells were plated in 96 well plate and incubated for $24 \mathrm{hr}$. Cells were then treated with CCTa siRNA and siRNA $S$ as indicated above. After $24 \mathrm{hr}, 20 \mu \mathrm{l}$ CTB reagent were added to the cells in each well and incubated for $1.5 \mathrm{~h}$. Fluorescence was then read at excitation/emission wavelengths of 560/590 nm in a Microplate Flourescence Reader FLx 800. For LDH assay, 50 $\mu$ l of supernatant obtained from cells grown under identical conditions as described in CTB assay were transferred from each well to a new 96 well plate. $125 \mu \mathrm{l}$ catalyst were added to $5925 \mu \mathrm{LDH}$ reagent and $50 \mu \mathrm{l}$ of the resulting LDH detection solution added to the supernatants. After 30 min incubation at room temperature in the dark; absorbance was read at a wavelength of $495 \mathrm{~nm}$ in a POWER X 340. Fluorescence and absorbance reading for $\mathrm{CTB}$ and $\mathrm{LDH}$ assays, respectively were also taken at 48 and 72 hours.

\section{Determination of cell death by acridine orange/ethidium bromide $(\mathrm{AO} / \mathrm{EB})$ staining}

$2.5 \times 10^{4} \mathrm{~A} 549$ cells were cultured in 8 well Lab-Tek II chamber slide and treated with siRNA S and CCTa-siRNA (400 nM) as described above. After incubation, cell death mechanism was determined by staining cells with $\mathrm{AO} / \mathrm{EB}$ and images viewed and captured by fluorescence microscope coupled with Olympus camera. 
Citation: Marijani R, Abonyo BO (2011) CTP: Phosphocholine Cytidyltransferase Alpha (CCTa) siRNA Induce Cell Death of Lung Cancer Cells. Pharm Anal Acta 2:121. doi:10.4172/2153-2435.1000121

Page 3 of 8

\section{Data analysis}

All data analysis was performed by GraphPad PRISM version 5.0 unless otherwise stated. Data is represented as mean $\pm \mathrm{SD}$. Where necessary, statistical analysis was performed by One Way Analysis of Variance (ANOVA) followed by Tukey's multiple comparison test.

\section{Results}

\section{CCTa siRNA sequences inhibit CCTa protein expression in A549 cells}

A549 cells were treated with various CCTa-siRNA and Western Blot analysis was used to determine expression of CCT $\alpha$ protein after these treatments. Figure 1 depicts results of Western Blot analysis as captured by FluorMax imager where all three sequences of CCT $\alpha$ inhibited protein expression even at a low concentration of 200nM. Compared to untreated cells, which exhibited strong bands, bands from CCTa siRNA samples were weak or completely disappeared. To show that the CCTa siRNA sequences were selective for CCTa protein inhibition, cells were also tested for inhibition of GAPDH gene. As shown in Figure 1, all transfected cell samples exhibited GAPDH protein bands consistent with untreated cells, indicating that the diminished or lack of CCTa protein expression was due to its inhibition by CCTa-siRNA sequences. Western blot band were further quantified by Quantity one to determine percent decrease densitometry in CCTa-siRNA treated cells. Densitometry ratios of CCT $\alpha$-siRNA samples were calculated relative to that of GAPDH. Figure $1 \mathrm{~A}$ depicts the quantification which shows significant decrease in CCT $\alpha$ expression when cells were treated with CCTa-siRNA. The densitometry percentage for treated cells averaged by 23,33 , and $4 \%$ for siRNA A, B, and C, respectively; with siRNA C showing the strongest inhibition of CCTa protein. This constitutes an average decrease of approximately $58-98 \%$ relative to GAPDH.

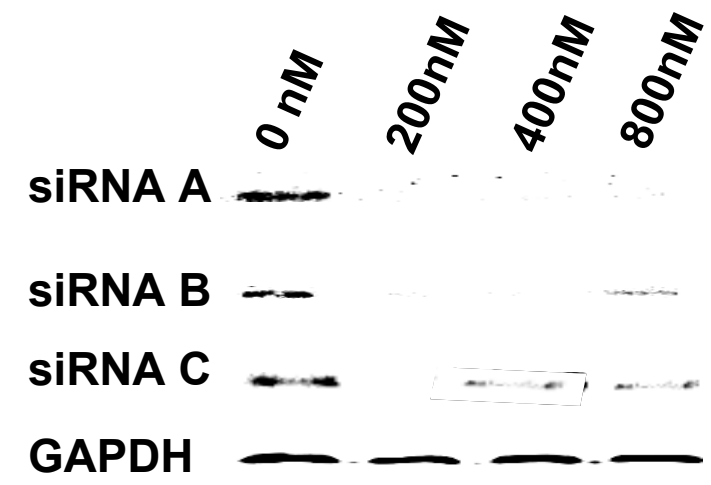

\section{CCTa-siRNA sequences induce A549 cell death}

CCT $\alpha$-siRNA and siRNA S treated cells as described above were further analyzed to determine whether the inhibition of CCTa protein which was confirmed by Western blot was causing cell death. After $24 \mathrm{hr}$ treatment, cells were visually observed under the microscope to study any morphological and structural changes that resulted from transfection. Figure $2 \mathrm{~A}$ represents images captured by the microscope showing that after $24 \mathrm{hrs}$, cells that untreated $(0 \mathrm{nM})$ and siRNA S treated cells remained intact with visible nuclei. However, addition of CCTa-siRNA caused cells to lose their morphology and intact structure deforming into granular structures indicative of apoptotic bodies; the process which progressed and caused total cell lyses as concentration of CCTa-siRNA increased. Additionally, the nuclei of the cells disappeared as the deformation occurred.

To validate these results, cells were transfected in 96 well plates and subjected to CTB and LDH assays. LDH assay is performed by assessing LDH released into the media as a marker of dead cells while CTB assay is a fluorometric method that estimates the number of viable cells in a cell population. CTB showed that untreated $(0 \mathrm{nM})$ cells exhibited an increase in fluorescence intensity (120) indicative of higher concentration of viable cells (Figure 2B). Addition of CCTasiRNA resulted into a significant reduction in fluorescence intensity from 120 to 42 for untreated and $100 \mathrm{nM}$ treated samples, respectively. A slight decrease in fluorescence intensity was observed when CCTasiRNA concentration was increased to $200 \mathrm{nM}$; however the reduction was not statistically significant. The decrease in fluorescence intensity is indicative of a decrease in number of viable cells. Further increase in CCTa-siRNA concentration did not produce significant changes in fluorescence intensity between 200 and $400 \mathrm{nM}$. LDH assay produced similar findings providing toxicity results which indicated low absorbance $(0.3 \mathrm{AU})$ for untreated cells, which increased $(0.76$ $\mathrm{AU}$ ) with addition of CCTa-siRNA (Figure 2C). All three sequences exhibited comparable results, suggesting that all were equally effective

\section{B}

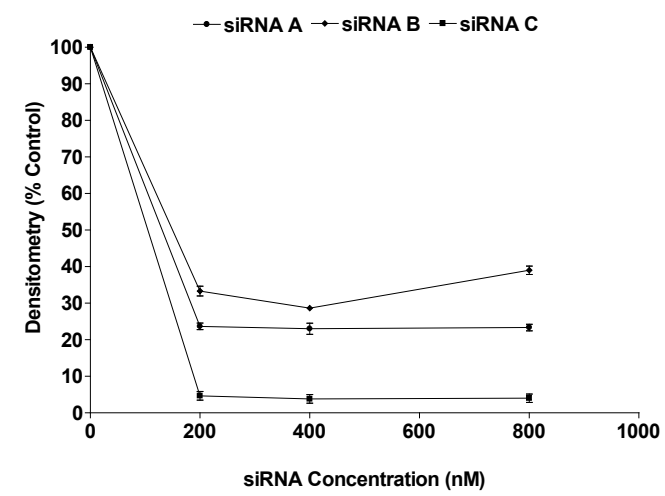

Figure 1: siRNA sequences inhibit CCTa protein expression in A549 cells. A549 cells cultured to $80 \%$ confluence were transfected with CCTa siRNA A, B and C $(0-800 \mathrm{nM})$, preincubated with FUGENE 6 transfection reagent at room temperature as indicated above. After 24 hr incubation, cell suspensions were centrifuged at $100 \mathrm{~g}$ and cell pellets resuspended in lysis buffer ( $20 \mathrm{mM}$ Tris, pH 7.4, $2 \mathrm{mM}$ EDTA, $150 \mathrm{mM} \mathrm{NaCl}, 0.5 \%$ Triton X-100 and one tablet/10 ml of protease inhibitor cocktail). Samples were sonicated for $3 \mathrm{~s}$, centrifuged $\left(5 \mathrm{~min}, 4^{\circ} \mathrm{C}\right.$ at $\left.1000 \mathrm{rpm}\right)$ and supernatants collected for total protein content analysis. Cell lysates were separated by

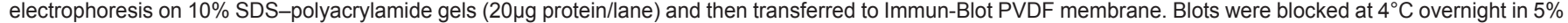
Carnation Instant Milk in PBS containing $0.05 \%$ Tween 20 (PBST) and then incubated overnight at $4^{\circ} \mathrm{C}$ with $5 \mu \mathrm{g} / \mathrm{ml}$ mouse anti-human CCT $\alpha$ affinity purified antibody. Membranes were washed three times with PBST, incubated with 1:1500 goat anti-mouse IgG-horse radish peroxidase in PBST for 3 hrs then washed three times in PBST. Immun-Blot images were obtained using a Flour-s Max Multimager. As shown in figure 1A, all the CCTa-siRNA sequences significantly inhibited CCTa protein expression with the disappearance of CCT $\alpha$ bands as CCT $\alpha$-siRNAs were added. Figure 1B shows corresponding reduced densitometry of CCT $\alpha$-siRNA treated sales as a ratio of GAPDH-siRNA. 
in inducing cell death. On the other hand, cells treated with siRNA $S$ showed an increase and decrease in fluorescence intensity and absorbance, respectively, which remained unchanged as concentration increased.

\section{A}

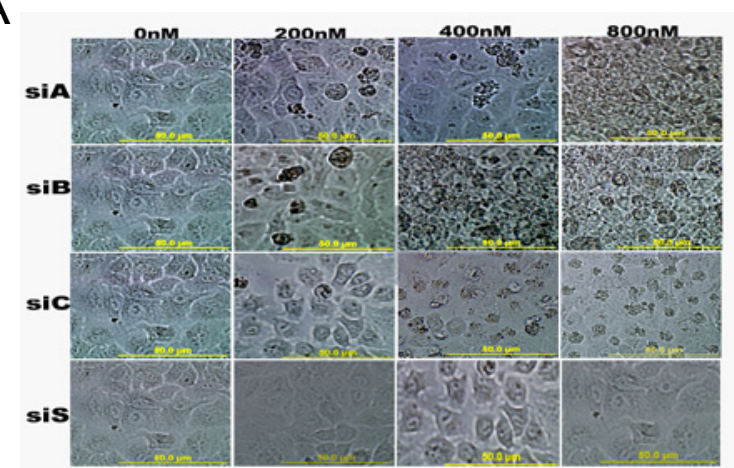

B

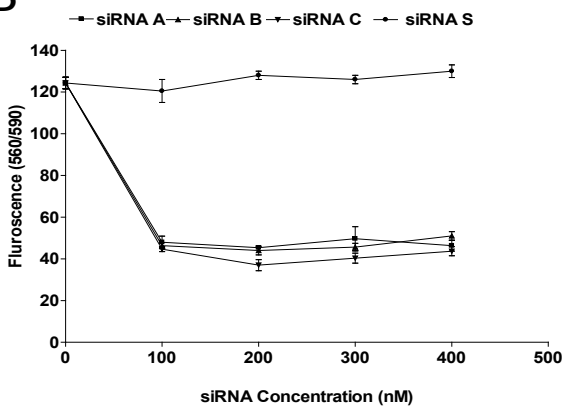

C

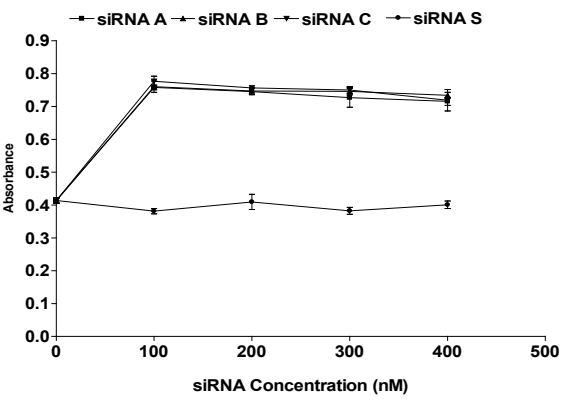

Figure 2: CCTa siRNA sequences induce A549 cell death. A549 cells cultured to $80 \%$ confluence were transfected with siRNA S and CCTa siRNA $\mathrm{A}, \mathrm{B}$ and $\mathrm{C}(0-800 \mathrm{nM})$, preincubated with FuGENE 6 at room temperature as indicated above. After $24 \mathrm{hr}$ incubation, cells were washed three times with PBS and incubated with Serum free media. Cell images were visualized and captured by an Olympus fluorescent microscope (40X objective) fitted with an Olympus DP70 camera. Images were documented using Adobe Photoshop. To determine cell viability, A549 cells were treated with different concentrations of CCTa-siRNAs in 96 well plates and living or dead cells detected by cell titre blue (CTB) or lactate dehydrogenase (LDH) cell viability assays, respectively. Briefly, cells treated with CCT $\alpha$ siRNAs in serum free media as indicated above were grown for $24 \mathrm{~h}, 20 \mu \mathrm{l}$ CTB reagent were afterwards added to the cells in each well. After a $1.5 \mathrm{~h}$ incubation, fluorescence was read at excitation/ emission wavelengths of $560 / 590 \mathrm{~nm}$ in a Microplate Foursecence Reader FLx 800. For LDH assay, 50 $\mu$ l of supernatant obtained from cells grown under identical conditions as described in CTB assay were transferred from each well to a new 96 well plate. $50 \mu \mathrm{l}$ of LDH detection solution added to supernatants. After 30 min incubation at room temperature in the dark, absorbance was read at a wavelength of $495 \mathrm{~nm}$ in a POWER X 340. As indicated in Figures 2A (Ligh microscopy), B (CTB) and C (LDH), CCTa siRNA induced cell death in A549 cells. Untreated and siRNA $S$ treated cells remained intact and exhibited no significant differences $(p>0.05)$ in the fluorescence intensity and absorbance, for CTB and LDH assay, respectively.

\section{CCT $\alpha$ siRNA sequences induce cell death in cells lacking p53}

To further validate the above results, CTB and LDH assays were conducted on p53 negative lung epithelial cell line (H1299) in order to rule out the presence and activity of p53 in A549 cells as a tumor suppressor and initiator of apoptosis. Figure $3 \mathrm{~A}$ represents Fluorescence intensity exhibited by CCT $\alpha$-siRNA treated H1299 cells; the intensity represents the amount of viable cells left after $24 \mathrm{hr}$ incubation. On the other hand, Figure $3 \mathrm{~B}$ depicts release of $\mathrm{LDH}$ as measured by absorbance; $\mathrm{LDH}$ release measures the extent of plasma membrane damage, which corresponds to cell death. Both CTB and LDH assays provided results similar to A549 cells where high fluorescence intensity (90) and low absorbance ( $0.45 \mathrm{AU})$ were observed with untreated cells, respectively. Moreover, fluorescence intensities and absorbencies of siRNA $S$ treated cells were not statistically significant between the concentration range. Addition of CCTa-siRNA consequently reduced fluorescence intensity to 50-60 with $100 \mathrm{nM}$ treatment. Slight increase occurred at $200 \mathrm{nM}$ for siRNA B and C, which dropped at $300 \mathrm{nM}$; however the change was statistically insignificant. Relative to untreated cells, the drop in fluorescence intensity for CCTa-siRNA treated cells ranged from 58.9-68.6, 47.8-59.3, and 51.3-63.2 \% for siRNA A, B, and $\mathrm{C}$, respectively. Similar findings were observed with $\mathrm{LDH}$ assay where addition of CCTa-siRNA resulted in approximately 43.8-73.6 \% increase in absorbance indicating increase in the amount of dead cells within the CCT $\alpha$-siRNA treated population. Further increase in CCTasiRNA concentration did not produce changes in the absorbance.

\section{Addition of PC did not reverse cell death induced by CCTa siRNAs}

A549 and H1299 cultured to $80 \%$ confluence were treated with PC $(100 \mu \mathrm{M})$ plus CCT $\alpha$ siRNA A, B and C or siRNA S (400 nM), preincubated with FuGENE 6 at room temperature processed for $\mathrm{CBT}$ assay as indicated above. Figure $4 \mathrm{~A}$ and $\mathrm{B}$ depict percentage fluorescence intensities as measured by the CTB assays for A549 and H1299 cell lines, respectively. From the figures we can see that addition of PC to CCT $\alpha$-siRNA transfected cells showed a decrease in fluorescence intensity indicating that more nonviable cells were available in these cells compared to untreated and siRNA $S$ treated cells. Relative to untreated cells, the decrease in fluorescence intensity was significant $(\mathrm{p}<0.001)$ and ranged from $34-61$ and $50-57 \%$ for A549 and H1299 cells, respectively. Slight decrease in fluorescence intensity [88\% (H1299) and 94\% (A549)] of siRNA S treated cells was observed compared to that of untreated cells, but the decrease was not statistically significant $(\mathrm{p}>0.05)$.

\section{Acridine orange/ethidium bromide (AO/EB) staining to detect apoptosis}

$\mathrm{AO} / \mathrm{EB}$ staining was used o visualize nuclear changes and apoptotic body formation that are characteristic of apoptosis. AO is a cationic dye that permeates selective nucleic acid, which can be taken up by both viable and nonviable cells and emits green fluorescence if intercalated into double stranded nucleic acid. EB intercalates and stains DNA, providing a red-orange fluorescence. Although it does not stain healthy cells, it can be used to identify cells that are in the early or final stages of apoptosis which have much more permeable membranes. Figure 5 exemplifies cell morphology as captured by Olympus camera. From this method, the extent of apoptosis was determined based on fluorescence emission and morphological aspect of chromatin condensation in stained nuclei. Results show that apoptotic cells had orange to red nuclei with condensed or fragmented chromatin, whereas viable cells had uniform bright green nuclei with intact structure; the latter results were observed with both the untreated and siRNA S treated cells. 
Citation: Marijani R, Abonyo BO (2011) CTP: Phosphocholine Cytidyltransferase Alpha (CCTa) siRNA Induce Cell Death of Lung Cancer Cells. Pharm Anal Acta 2:121. doi:10.4172/2153-2435.1000121

\section{Discussion}

It is evident from reported literature that the use of siRNA for targeted gene knockdown has provided advantageous results relative to conventional therapies. It reported that design and application of androgen receptor (AR) siRNA successfully knocked down AR and AR protein expression, which led to dramatic prostate cancer cell death in vitro and inhibition of prostate tumor growth in vivo [26]. Another study by Cioca et al. [28] reported a siRNA approach that knocked down Raf-1 kinase and Bcl-2 oncoprotein in myeloid leukemia cell lines. The outcome of these and many other studies [26-29] have provided the insight and potential of siRNA application in designing new cancer therapies. However, there are still shortcomings in this approach that have to be addressed, most importantly the specificity of the siRNAs as many have shown to damage both cancerous and normal cells. Additionally, available reports provide information of studies that

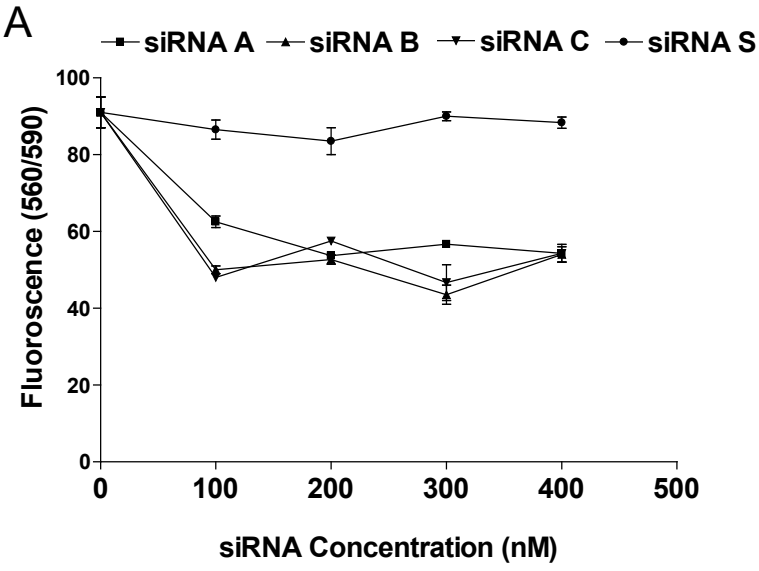

B

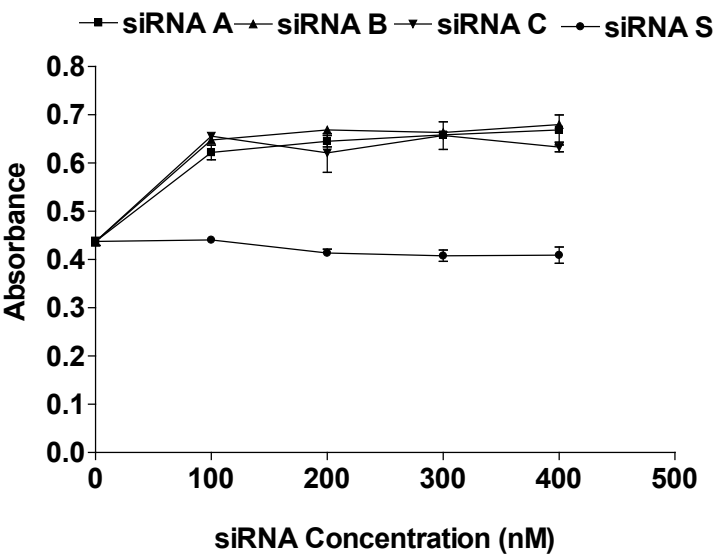

Figure 3: CCT $\alpha$ siRNA sequences induce cell death cell lines lacking p53. H1299 (lung carcinoma cells that lack expression of p53 protein) cultured to $80 \%$ confluence were transfected with siRNA S and CCTa siRNAs A, B and $\mathrm{C}(0-400 \mathrm{nM})$, preincubated with FuGENE 6 at room temperature as indicated above. Cells were processed for imaging, CTB and LDH assays as indicated above. Light microscopy (Figure not shown), CTB assay (Figure 3A) and LDH assay (Figure 3B) demonstrated that CCTa siRNA induced death in cells lacking p53. On the contrary cells that were treated with siRNA S did not show significant changes in intensity fluorescence and absorbencies.
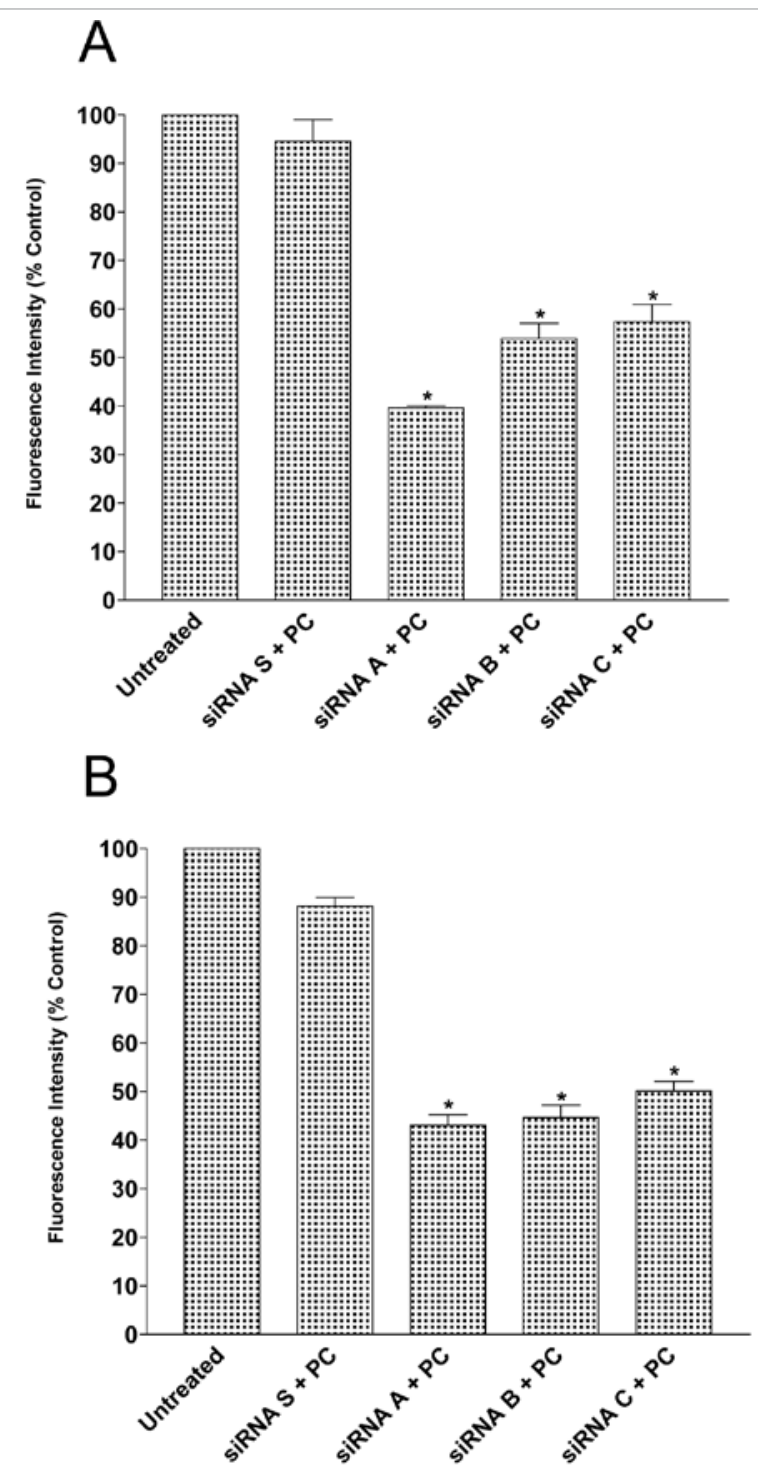

Figure 4: Addition of Phosphatidylcholine did not reverse cell death induced by CCT $\alpha$ siRNAs. A549 and $\mathrm{H} 1299$ cultured to $80 \%$ confluence were transfected with siRNA S and CCT $\alpha$ siRNA A, B and C $(0-400 \mathrm{nM})$, preincubated with FuGENE 6 plus $100 \mu \mathrm{M}$ PC at room temperature. After treatment, cells were incubated at $37^{\circ} \mathrm{C}$ for $24 \mathrm{hr}$ and processed for CTB assay as indicated above. Figure 4 represents percent reduction in fluorescence intensity for A549 and $\mathrm{H} 1299$ cells, respectively, relative to untreated cells. Significant differences $(p<0.01)$ were seen in the reduction of CCTa-siRNA + PC treated cells for both A549 (A) and H1299 (B) cells. No significant differences were observed between concentrations of siRNA S + PC treated cells. Data is presented as average of three experiments $\pm S D$. *indicates significant changes relative to untreated cells.

have targeted genes that are specific for a particular cancer; therefore finding and establishing a siRNA that will be applicable and target gene (s) expressed in all cancers will be valuable to the development of new cancer therapies.

Our current experiments and results provide compelling support that inhibition of CCTa protein by CCTa-siRNAs cause cell death of A549 and H1299 cells. Previous works by Baburina and Jackowski, [7] reported cytotoxicity activity of ET-18-OCH3 compound which targeted CCT. Additionally, the investigated compound was able to inhibit CCT activity in vitro by competing with lipid activator site on the 
enzyme. Results from their study strongly support that CCT inhibition was the cause of ET-18-OCH3 induced apoptosis. Our results however, emphasize the specific inhibition of CCTa protein expression, which is evidenced by the Western blot analysis in which substantial decrease in CCT $\alpha$ protein expression is observed when A549 cells were treated with various concentrations of CCTa-siRNA. As an internal control GAPDH or scrambled siRNA, did not compromise CCTa protein expression. These results affirm that CCT $\alpha$-siRNA was specific to inhibition of CCTa protein. The relationship between inhibition of CCTa protein and cell death was exemplified by the cell viability and toxicity assays that showed consequently that lack of CCTa could induce cell death. Cell titer blue assay provides a fluorometric measure of viable cells available in a population. The assay is based on the ability of viable cells to convert resuzurin redox dye into resorufin, which is fluorescent; therefore, the higher the fluorescence intensity the more viable the cells. In this case, nonviable cells lose their capability of reducing resuzurin into resorufin due to lack or metabolic capacity. As seen in our results, lack of CCTa protein which plays a crucial role in one of the major pathways in eukaryotic cell membranes, compromised the cells resulting into cell death. The inhibition of CCTa gene by CCT $\alpha$ siRNAs may have attributed to the reduced or loss in viable cells as measured by CTB assay. To confirm the results we used the LDH assay, which measured membrane integrity as an indication of cell damage by monitoring LDH release into the cytoplasm. Typically, membranedamaged cells would release LDH that will convert tetrazonium salt to formazan dye, which is directly proportional to the number of dead or damaged cells. This was found to be the case with our CCTa-siRNA treated cells as they showed increased absorbance which was used to measure the release of formazan dye corresponding to the amount of dead cells in the population. Both assays in their respective aspects assert that there is a compromise in cell growth when CCTa-siRNAs are added to the cells and we can safely say that the compromise is a result of inhibition of CCTa gene expression as shown by Western blot. Additionally, by exhibiting similar results in H1299 cells, which lack p53 gene, we rule out the contribution of p53 in A549 cells as an initiator of apoptosis. However, with these results, further investigation was needed to explore the involvement of CCT $a$ inhibition in relation to $\mathrm{PC}$ as a cause of apoptosis induction.

A more wider/general target on gene knockdown in cancer research has been the CDP-Choline pathway, which is the primary route to PC synthesis; a crucial phospholipid in eukaryotic membrane structure. Many studies have addressed and reported targeting inhibition of PC synthesis as a means of inducing cell death. Different approaches have been taken to chemically inhibit PC synthesis. Reports have provided insightful outcomes [30-32], that some have led to development of chemical anti cancer and cytotoxic drugs such as ET-18-OCH3, HexPC, and farnesol that target PC synthesis. Zweigner et al. [33] showed bacterial infection induced cell death of brain cells through inhibition of PC synthesis. They further reported that addition of lyso-PC to infected cells and culture medium before injection prevented human cell toxicity and apoptosis compared with untreated cells. On a different approach, deprivation of PC has also been found to cause cell death of PC 12 neuronal cells, where cells that contained serum or choline with serum free medium grew rapidly for over five days. However, cells that were grown in serum free medium without choline stopped proliferating [34]. These studies and several others have explored potential benefits in targeting the CDP-Choline

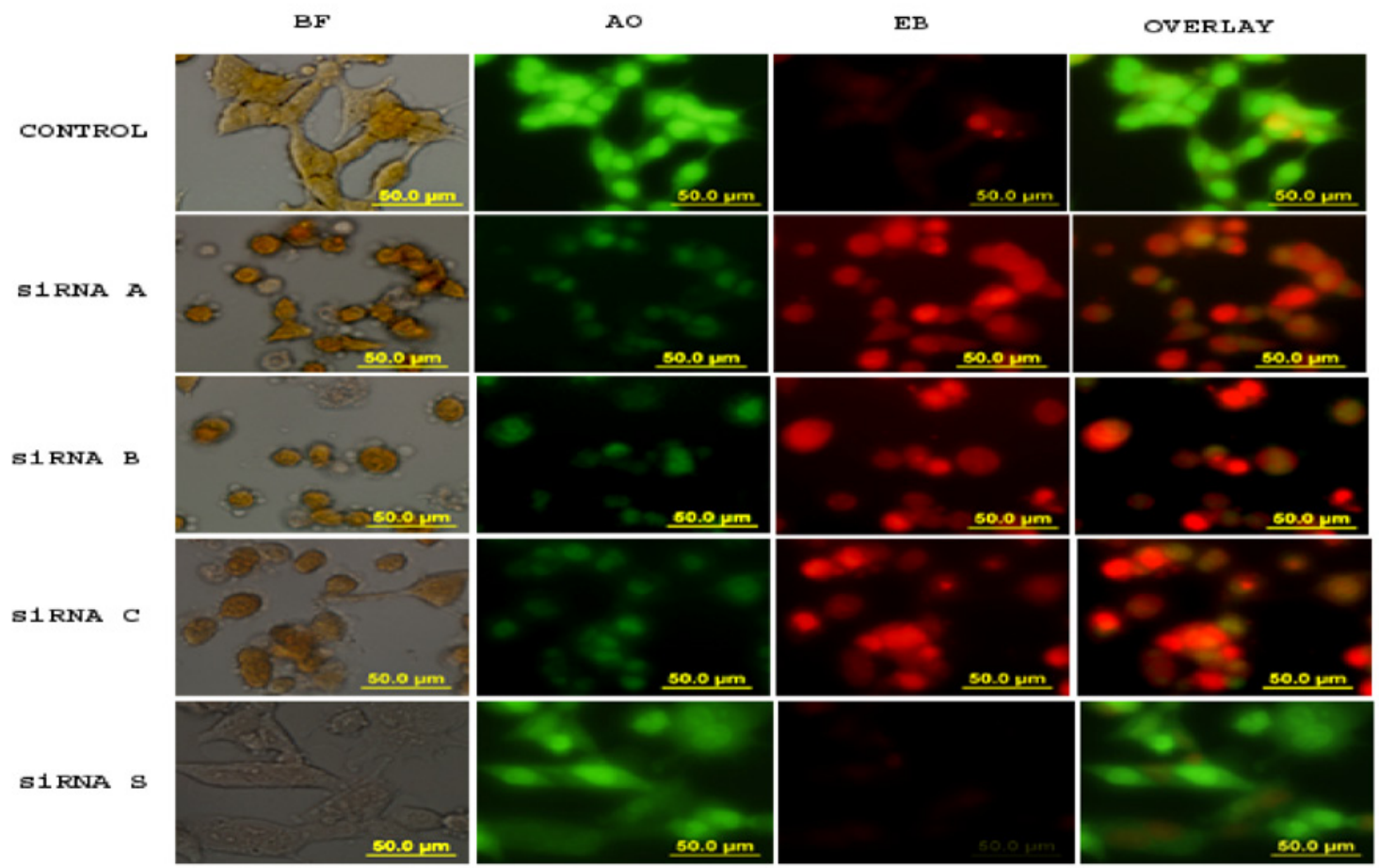

Figure 5: Apoptosis is evidenced by AO/EB double staining. Morphological study of A549 cells cultured in 8 well Lab-Tek II chamber slide and treated with $400 \mu \mathrm{M}$ of various sequences of CCTa-siRNA: siRNA A, B, and C and siRNA S for $24 \mathrm{hr}$. The figure represents images viewed and captured by Fluorescence microscope coupled with Olympus camera. After incubation, cells were stained with AO/EB to detect apoptosis. Images were captured at different settings, BF: Bright Field; AO Acridine Orange; EB: Ethidium Bromide. The last column represents the overlay images of AO and EB. Viable cells excluded ethidium bromide and their nuclei were bright green with intact structure, while apoptotic cells were orange to red color with highly condensed nuclei. 
pathways and attempted to investigate its relationship with apoptosis. The CDP-Choline pathway involves 3 Steps in its production of PC; another plausible approach is targeting the rate limiting enzyme, CCT. One of the major isoforms of CCT, CCTa has shown to play a central role in CDP-choline pathway exhibiting crucial involvement in the production of PC. This factor may attribute to the substantial cell death caused by knockdown of this protein using siRNA. Moreover, in the current investigation, CCTa-siRNA treated cells failed to recover after PC was added suggesting potential failed recovery of cancer cells when targeted by CCT $\alpha$-siRNA. Furthermore, observation of cells treated with a scrambled sequence siRNA showed that all treatments maintained their structure and viability, confirming that the death was specifically caused by CCT $\alpha$-siRNA.

Apoptosis is a natural method by which human body gets rid of defective cells in a localized manner. On the contrary, killing cells by necrosis induce release of cell components that may trigger potent inflammatory reactions in the surrounding tissues. In the current investigations, we determined that the use of lower concentrations of CCTa-siRNA resulted in cell death by apoptosis. However, high concentrations induced cell lysis. In this context it appears that the use of lower concentrations of CCTa-siRNA will be appropriate. Determination that CCT inhibition results in cell death by apoptosis is not unique to our investigations. Several investigators have reported that significant inhibition of PC synthesis in cells results apoptosis [3537]. The resulting apoptosis has also been shown to be partially reversed by addition of PC or lysophasphatidylcholine supplements [37]. What is new in this current investigation is that the apoptosis is induced by CCT $\alpha$-siRNA and that appropriate concentration for specific method of cell death can be designed.

The present work investigated the potential of CCT $\alpha$-siRNA to induce cell death to lung epithelial cancer cells. Our study focused on the CCT $\alpha$ isoform as it has been reported to be more critically involved in cell development compared to its CCT $\beta$ counterpart. Currently, there are no reports on the use of CCTa-siRNA and apoptosis. Although studies have shown that apoptosis can result from chemical inhibition of CCTa, these approaches have the potential of high level toxicity. Additionally, reports are still conflicting as to whether the apoptosis is as a result of PC depletion which is caused by CCTa inhibition. In the current study, the CCTa-siRNA was used to directly knockdown this critical enzyme. The results presented here demonstrate the ability of various sequences of CCT $\alpha$-siRNA to inhibit CCT $\alpha$ protein expression, which consequently caused cell death in both p53 positive and negative lung cancer cells.

\section{Acknowledgments}

Support for this research was provided in part by NIH grants 1R15HL09371001A1 and NIH/NCRR/RCMI G12RR03020.

\section{References}

1. Cui Z, Houweling M (2002) Phosphatidylcholine and cell death. Biochim Biophys Acta 1585: 87-96.

2. Ruiz-Cabello J, Cohen JS (1992) Phospholipid metabolites as indicators of cancer cell function. NMR Biomed 5: 226-233.

3. Aboagye EO, Bhujwalla ZM (1999) Malignant transformation alters membrane choline phospholipid metabolism of human mammary epithelial cells. Cancer Res 59: 80-84.

4. Tessner TG, Rock CO, Kalmar GB, Cornell RB, Jackowski S (1991) Colonystimulating factor 1 regulates CTP: phosphocholine cytidylyltransferase mRNA levels. J Biol Chem 266: 16261-16264.

5. van der Sanden MH, Houweling M, van Golde LM, Vaandrager AB (2003) Inhibition of phosphatidylcholine synthesis induces expression of the endoplasmic reticulum stress and apoptosis-related protein CCAAT/enhancerbinding protein-homologous protein (CHOP/GADD153). Biochem J 369: 643650 .

6. Voziyan PA, Goldner CM, Melnykovych G (1993) Farnesol inhibits phosphatidylcholine biosynthesis in cultured cells by decreasing cholinephosphotransferase activity. Biochem J 295: 757-762.

7. Baburina I, Jackowski S (1998) Apoptosis Triggered by 1-O-Octadecyl-2-Omethyl-rac-glycero-3-phosphocholine is prevented by increased expression of CTP:Phosphocholine Cytidlyltransferase. The $\mathrm{J}$ of Biological Chemistry 273 2169-2173.

8. Karim M, Jackson P, Jackowski S (2003) Biochim Gene structure, expression and identification of a new CTP: phosphocholine cytidylyltransferase beta isoform. Biophys Acta 1633: 1-12.

9. Veitch DP, Gilham D, Cornell RB. (1999) The role of histidine residues in the HXGH site of CTP:phosphocholine cytidylyltransferase in CTP binding and catalysis. Eur J Biochem 255: 227-234.

10. Veitch DP, Cornell RB (1996) Substitution of serine for glycine-91 in the HXGH motif of CTP: phosphocholine cytidylyltransferase implicates this motif in CTP binding. Biochemistry 35: 10743-10750.

11. Park YS, Gee P, Sanker S, Schurter EJ, Zuiderweg ERP, et al. (1997) Identification of functional conserved residues of CTP: glycerol-3phosphatecytidylyltransferase. J Biol Chem 272: 15161-15166.

12. Wang Y, MacDonald JIS, Kent C (1995) Identification of the nuclear localization signal of rat liver CTP: phosphocholine cytidylyltransferase. J Biol Chem 270 : 354-360.

13. Yang W, Boggs KP, Jackowski S (1995) The association of lipid activators with the amphipathic helical domain of CTP: phosphocholine cytidylyltransferase accelerates catalysis by increasing the affinity of the enzyme for CTP. J Bio Chem 270: 23951-23957.

14. Lykidis A, Baburina I, Jackowski S (1999) Distribution of CTP:phosphocholin cytidylyltransferase (CCT) isoforms. Identification of a new CCT $\beta$ splice variant. J Biol Chem 274: 26992-7001.

15. Wang Y, Sweitzer TD, Weinhold PA, Kent C (1993) Nuclear localization of soluble CTP: phosphocholine cytidylyltransferase. J Biol Chem 268: 58995904

16. Weber CH, Park YS, Sanker S, Kent C, Ludwig ML (1999) A prototypical cytidylyltransferase: CTP: glycerol-3-phosphate cytidylyltransferase from Bacillus subtilis. Structure 7:1113-1124.

17. Yang W, Jackowski S (1995) Lipid activation of CTP: phosphocholine cytidyly -transferase is regulated by the phosphorylated carboxyl-terminal domain. $J$ Biol Chem 270: 16503-16506.

18. Jackowski S, Rehg JE, Zhang, YM, Wang J, Miller K, et al. (2004) Disruption of CCTbeta2 expression leads to gonadal dysfunction. Mol Cell Biol 24: 4720 4733

19. Wang L, Magdaleno S, Tabas I, Jackowski S (2005) Early embryonic lethality in mice with targeted deletion of the CTP: phosphocholine cytidylyltransferase alpha gene (Pcyt1a). Mol Cell Biol 25: 3357-3363.

20. Friesen JA, Campbell HA, Kent C (1999) Enzymatic and cellular characterization of a catalytic fragment of CTP: phosphocholine cytidylyltransferase alpha. J Biol Chem 274: 13384-13389.

21. Lykidis A, Jackowski S (2001) Regulation of mammalian cell membrane biosynthesis. Prog Nucleic Acid Res Mol Biol 65: 361-93.

22. Lykidis A, Jackson P, Jackowski S (2001) Lipid activation of CTP:phosphocholine cytidylyltransferase a: characterization and identification of a second activation domain. Biochemistry 40: 494-503.

23. Zhou J, You Y, Ryan JA, Mallampalli KR (2004) Upregulation of surfactant synthesis triggers ABCA1-mediated basolateral phospholipid efflux. Journal of Lipid Research 45: 1758-1767.

24. van der Luit AH, Budde M, Ruurs P, Verheij M, van Blitterswijk WJ (2002) Alkyl-lysophospholipid accumulates in lipid rafts and induces apoptosis via raftdependent endocytosis and inhibition of phosphatidylcholine synthesis. J Biol Chem 277: 39541-39547.

25. Ramos B, El Mouedden M, Claro E, Jackowski S (2002) Inhibition of CTP:Phosphocholine Cytidylyltransferase by C2-Ceramide and Its Relationship to Apoptosis Molecular phamacolody. 62: 1068-1075. 
Citation: Marijani R, Abonyo BO (2011) CTP: Phosphocholine Cytidyltransferase Alpha (CCTa) siRNA Induce Cell Death of Lung Cancer Cells. Pharm Anal Acta 2:121. doi:10.4172/2153-2435.1000121

26. Liao X, Tang S, Thrasher JB, Griebling TL, Li B (2005) Small-interfering RNAinduced androgen receptor silencing leads to apoptotic cell death in prostate. Mol Cancer Ther 4: 505-515.

27. Zegarra-Moro OL, Schmidt LJ, Huang H, Tindall DJ (2002) Disruption of androgen receptor function inhibits proliferation of androgen-refractory prostate cancer cells. Cancer Res 62: 1008-1013.

28. Cioca DP, Aoki Y, Kiyosawa K (2003) RNA interference is a functional pathway with therapeutic potential in human myeloid leukemia cell lines. Cancer Gene Ther 10: 125-133.

29. Eder IE, Culig Z, Ramonor R (2000) Inhibition of LNCaP prostate cancer cells by means of androgen receptor antisense oligonucleotides. Cancer Gene Ther 7: $997-1007$

30. Ruiter GA, Verheij M, Zerp SF, van Blitterswijk WJ (2001) Alkyllysophospholipids as anticancer agents and enhancers of radiation-induced apoptosis. Int J Radiat Oncol Biol Phys 49: 415-419.

31. Bladergroen BA, Bussierre M, Klein W, Geelen MJH, van Golde LMG, et al. (1999) Inhibition of phosphatidylcholine and phosphatidylethanolamine biosynthesis in rat-2 fibroblasts by cell-permeable ceramides. Eur J Biochem. 264: $152-160$.
32. Allan D (2000) Lipid metabolic changes caused by short-chain ceramides and the connection with apoptosis. Biochem J 345: 603-610.

33. Zweigner J, JAckowski S, Smith S (2004) Bacterial inhibition of Phosphatidylcholine syhethsis triggers apoptosis in the brain. J Exp Med 200: 99-106.

34. Holmes-McNary MQ, Loy R, Mar M-H, Albright CD, Zeisel SH (1997) Apoptosis is induced by choline deficiency in fetal brain and in PC12 cells. Dev Brain Res 101: $9-16$

35. Boggs KP Rock CO Jackowski S (1995) Lysophosphatidylcholine and 1-O-octadecyl-2-O-methyl-rac-glycero-3-phosphocoline inhibit the CDPcholine pathway of phosphatidylcholine synthesis at the CTP:phosphocjoline cytidylyltransferase step. J Niol Chem 270: 7757-7764.

36. Boggs KP Rock CO Jackowski S (1995) Lysophosphatidylcholine attenuate the cytotoxic effects of the antineoplastic phospholipid 1-O-octadecyl-2-Omethyl-rac-glycero-3-phosphocoline. J Biol Chem 270: 11612-11618.

37. Haug JS, Goldner CM, Yazlovitskaya EM, Voziyan PA, Melnykovych G (1994) Directed cell killing (apoptosis) in human lymphoblastoid cells incubated in the presence of farnesol: effect of phosphatidylcholine. Biochim Biophys Acta 1223: $133-140$ 